\title{
Professional Interactions: Negotiation and Expression for Future Physicians and Healthcare Providers
}

\author{
Andrew J. Pan ${ }^{1, *}$, Aaron J. Pan², Kimberlyn R. Leary ${ }^{3,4,5,6}$ \\ ${ }^{1}$ School of Medicine and Dentistry, University of Rochester, Rochester, 14642, New York, United States \\ ${ }^{2}$ New York Chiropractic College, Seneca Falls, 13148, New York, United States \\ ${ }^{3}$ The National Math and Science Initiative, Dallas, 75206, Texas, United States \\ ${ }^{4}$ Department of Health Policy and Management, Harvard Chan School of Public Health, Boston, 02115, Massachusetts, United States \\ ${ }^{5}$ Enabling Change Program, Harvard Chan School of Public Health, Boston, 02115, Massachusetts, United States \\ ${ }^{6}$ Department of Psychiatry \& McLean Hospital, Harvard Medical School, Boston, 02115, Massachusetts, United States
}

Copyright $\bigcirc 2017$ by authors, all rights reserved. Authors agree that this article remains permanently open access under the terms of the Creative Commons Attribution License 4.0 International License

\begin{abstract}
The rapid pace of change in medicine requires doctors to be effective conflict mediators and negotiators in the clinical workplace, and a multitude of research connects strong physician-patient communication to improved patient outcomes. Disparities in such skills exist among medical students and professionals, and are neither taught nor evaluated in a standardized fashion in U.S. medical school curriculums. A structured course would benefit patients by providing protected time for medical students to refine these skills. Our proposed course, titled "Professional Interactions: Negotiation and Expression for Future Physicians and Healthcare Providers" and referred to hereafter as PINE, is outlined here to provide a starting point for those seeking to create or supplement a similar course at their institutions. PINE, ideally, would tap the multidisciplinary expertise of leaders in medicine, the humanities, business, local community organizations, and education to facilitate student engagement with a variety of topics in interpersonal communication and negotiation.
\end{abstract}

Keywords Problem-solving, Negotiation, Communication, Conflict Resolution, Inter-professionalism

\section{Introduction}

Effective communication, in its many forms, is a requirement for success in virtually every profession, including healthcare. Research suggests that there are discrepancies in communication skills among providers, which contribute to lack of patient engagement, medical errors, and potentially malpractice suits [1-3]. Likewise, there is a wealth of literature tying effective communication and interaction to improved health outcomes and quality measures such as adherence to treatment, satisfaction ratings, patient participation in care, and self-management [4-9].
Fundamentally, strong communication facilitates delivery of patient-centered care, integrating biomedical management of a patient's disease with attention to social, cultural, and other aspects of the patient [10-12] to provide better treatment [13].

In addition to effective communication, patient-centered care also entails problem solving, an equally important skill area in medicine. For the purposes of PINE, problem solving will refer specifically to negotiation, which, in turn, encompasses stewardship. "Stewardship" was the term used in an AMA Viewpoints post [14] to specifically describe the responsibility of providing the best patient care through prudent use of limited resources. On a broader level, situations in which medical advice, patient viewpoints, and administrative goals may differ are opportunities for negotiation and conflict transformation [15], which in turn may help patients and providers strengthen their relationships with one another. With the advent of healthcare reform, managed care, the "employed physician," and other changes, American medicine has become increasingly complex and interdisciplinary. Future physicians can expand their capacity for effective practice when they are well-rounded professionals [16-18] and problem-solvers in both scientific and non-scientific contexts.

\section{Significance}

As of this writing, communication and negotiation courses are neither official medical school admissions requirements nor medical school curriculum components [19]. Current means of evaluating these skills in applicants, which include brief essays, traditional interviews, and even Multiple Mini Interview stations, remain subjective, limited, situational, and inconsistent. This contrasts with evaluation of student knowledge for arguably less-used disciplines such as physics and organic chemistry, which are standard academic 
prerequisites and longstanding features of the Medical College Admission Test ${ }^{\circ}$ (MCAT).

Once accepted to medical school, students complete preclinical coursework that may only sporadically reference those skills in isolated lectures or activities, if at all. Current clinical exams and patient exposure experiences provide standardized testing for core clinical skills and maneuvers, but do not test or establish organized, bottom-up communication or negotiation skill sets that can be adapted and utilized in a healthcare setting.

Thus, it is not surprising that there can be striking discrepancies of communication and negotiation ability among students and healthcare providers. Given that communication skills actually worsen for students throughout medical school in the absence of training [20], and that patients are even more concerned with physician expression of care than with knowledge [21], it is clear that a reflective, longitudinal means of developing and assessing communication and negotiation skills is warranted.

\section{Description}

While changes in undergraduate coursework or medical school admissions requirements are possible solutions, our focus here is on medical students, who have already been screened by admissions committees for demonstrated proficiency in the basic sciences. We propose our course, "Professional Interactions: Negotiation and Expression for Future Physicians and Healthcare Providers" (PINE) to provide opportunities for structured learning time for physicians-in-training to develop strong communication and problem-solving skills. Such a course would add educational value through both its content and longitudinal arrangement, allowing for continual reinforcement and formal evaluation during the preclinical and clinical years of medical school. The course's strengths would stem from a powerful combination of diversely sourced learning materials, inter-professional expertise and learning opportunities, and an active, engaging teaching and learning environment. Principles of communication and negotiation will be taught with a focus on application in clinical contexts, with the communication component ideally preceding the negotiation component to provide an appropriate foundation for the latter. Provided below are suggestions for PINE's curriculum and logistics.

\section{Learning Objectives}

At the end of this course, students will be able to:

- Identify disparate styles of communication and gain insight on their preferred styles, to be able to adapt as future clinical situations require.

- Understand and apply basic negotiation principles and problem-solving frameworks to defuse conflicts and strengthen relationships with patients and colleagues.
Analyze and evaluate the conditions that foster team effectiveness, the factors that can disrupt team performance, and the system features that enable temporary, emergent teams to coordinate problem-solving approaches.

- Become strong interviewers who can also take a medical history consistent with their level of training.

- Write and speak at a level consistent with a professional career.

- Effectively present, explain, and teach medical and scientific concepts to others with similar and dissimilar backgrounds.

- Efficiently navigate research databases and online healthcare references, and be able to distinguish and discuss misinformation with patients.

- Gain awareness of the medical, cultural, and other beliefs of those from diverse backgrounds, and consider these in the contexts of communicating and negotiating as a healthcare provider.

\section{Communication}

Lecture 1: The Road Ahead

- Course Introduction/Logistics

- Understanding Your Personal Style: Analytical, Intuitive, Functional, Personal (Quiz) [22]

Lecture 2: Written Expression

- Professional emails and letter writing

- Effective Phrasing vs. colloquialisms

- In-class workshop/homework

Lecture 3: Digital Communication I

- Intro to patient confidentiality

- Intro to electronic health records

- Intro to telemedicine

Lecture 4: Digital Communication II

- Medicine and social media

- Effective use of medical apps

- Medical blogging/journalism

- Pharma advertising to doctors and patients

Lecture 5: Digital Communication III

- Using and searching literature databases and e-libraries within institutions

- Assessing source validity and accuracy, and discussing misinformation with "web-savvy patients"

Lecture 6: Visual and Pedagogic Expression

- Elements of an effective presentation, with focus on presenting research or lectures on medical topics (builds off Lectures 2 and 5)

- Elements of effective visuals

- Sample grand rounds or conferences (videos/demos; 
encouraging students to sit in on grand rounds as schedules permit)

Lecture 7: Patient Interviews I: Transition

- Basic Interviewing skills (non-medical)

- Establishing trust; building rapport

- Subtle Language: Non-verbal cues (tone, dress, body language, etc.)

- Giving and receiving appropriate feedback and criticism

- Practice interview sessions/scenarios

Lecture 8: Patient Interviews II: The Basics

- Elements of medical history taking

- Explaining medical jargon and complex diseases or conditions to patients (builds off Lecture 6)

- SOAP note-writing (builds off Lecture 2)

- Standardized patient practice sessions (builds off Lecture 7)

Lecture 9: Patient Interviews III: Sensitivity

- Asking about sensitive topics (drug use, sexual activity, etc.)

- Delivering bad news; showing empathy

- Videos, practice cases

- Standardized patient practice sessions

Lecture 10: Patient Interviews IV: Difficulty

- Methods for Difficult Patients

- Methods for Hostile or Unethical Situations

- Videos, practice cases

- Standardized patient practice sessions

Lecture 11: Patient Interviews V: Diversity

- Communication nuances across cultures, genders, ages, socioeconomic conditions

- Special services (foreign language, blind, deaf interpretation; communicating with mentally challenged patients)

- Standardized patient practice sessions

Lecture 12: Allies in Healthcare

- Communicating within a hierarchy (direct superiors, hospital management, etc.)

- Communication across a team (other physicians, allied health providers, etc.); effective hand-offs

- Practice cases for interprofessional teams of medical, nursing, PA, and other professional students

\section{Negotiation}

Lecture 13: Getting to Yes

- Intro to principles of negotiation and conflict resolution

- Distributive bargaining

- Mutual gains model

- Identifying one's preferred styles
- Assigned reading: Fisher and Ury's Getting to Yes

Lecture 14: Working with Emotions

- Understanding and utilizing emotions in negotiation

- Reaching mutual understanding; psychology of rapport-building (builds off Lecture 7)

- Pre-assigned videos or cases; in-class simulations

- Assigned reading: Fisher and Shapiro's Beyond Reason

Lecture 15: Negotiating with Numbers

- Principles of bargaining

- Intro to health economics

- Insurance claims, salaries, contracts, etc.

- Pre-assigned videos or cases; in-class simulations

Lecture 16: Negotiating with Values and Beliefs

- Intro to Bioethics

- Common ethical and other dilemmas for healthcare professionals

- Nuances of different belief systems (awareness of different cultural/religious views on aspects of medicine and how to work with them)

- Pre-assigned videos or cases; in-class simulations

Lecture 17: Negotiating with Power Differentials

- Builds off Lecture 10

- Working effectively with superiors

- Methods for handling a hostile workplace

- Difficult Patients (hostility; nonadherence; political or economic leverage, etc.)

- Pre-assigned videos or cases; in-class simulations

Lecture 18: Negotiating with Power Differentials II

- Builds off Lecture 11

- Intro to patient advocacy

- Nuances of diverse backgrounds (impact of culture, gender, age, socioeconomics, education, etc. on negotiation)

- Contemporary cross-cultural issues

- Awareness of local community outreach efforts and social resources

- Pre-assigned videos or cases; in-class simulations

Lecture 19: Leadership and Teamwork

- Builds off Lecture 12

- Principles of Leadership

- Team dynamics, inter-professionalism, shared or group decision-making)

- Pre-assigned videos or cases; in-class simulations

Lecture 20: Improvisation

- Improvisation/acting exercises

- Pre-assigned videos or cases; in-class simulations

Lecture 21: Negotiating Using Technology

- Dynamics of communicating over webcam, phone, 
or instant messaging

- Pre-assigned videos or cases; in-class simulations

\section{Possible Lecturers}

- Medical faculty

- Research faculty/medical librarians

- Humanities faculty (communication, ethics topics)

- Hospital ethics committee members

- Hospital chaplains

- Speaking coaches, journalists, app developers, etc.

- Business/law faculty for negotiation and economics topics

- Community leaders (cultural, religious, etc.) and social advocates for diversity and outreach topics

\section{Possible Materials}

- Primary literature

- Books (negotiation or communication texts)

- Required negotiation text: Ury's Getting to Yes; Ury's Getting to Yes with Yourself; Chris Anderson's TED Talks

- Required communication texts: Beebe and Mottet's Business \& Professional Communication: Principles and Skills for Leadership; Zoller and Preston's You Said What?!: The Biggest Communication Mistakes Professionals Make (A Confident Communicator's Guide)

- Other supplemental texts per instructor discretion

- Online articles

- Multimedia resources

- Negotiation simulations and exercises

- Case studies from Harvard Business Publishing, etc.

- Videos

- Standardized patient vignettes or practice cases

- White coat pocket cards with negotiation and communication reminders

\section{Possible Activities}

- Guest lectures

- Small group activities

- Discussions

- Workshops

- Problem-based learning cases (PBL)

- Practice interviews

- Inter-professional sessions

- Practice individual interviews

- Standardized Patient interviews/demos

\section{Possible Assessments}

- Short, multiple choice assessments with NBME®-style clinical vignettes
- Many-on-one (students with faculty) assessment interviews or simulations

- Videotaped for review, with immediate feedback sessions and reflection surveys

- Skill-area rubrics for pass/fail evaluation

- Possible use of modified checklists used for current clinical medicine courses

- $\quad$ Targeted Evaluation forms

- Faculty-student as well as student-student end-of-course evaluations for ongoing improvement

- Teach-back presentations

- For example, presentation skills could be demonstrated via "teach-back" lectures on concurrent basic science content for first year students or review lectures in Year 2 for new first year students

\section{Possible Grading Scheme}

- We recommend for this course to be designated pass/fail as most medical students' efforts should still be devoted to their basic science coursework.

- We recommend for $30 \%$ of points to be from multiple choice assessment and assignments, and $70 \%$ of points to be from final simulations assessment evaluations. Students must score $70 \%$ of total points to be eligible to pass the course. We leave the specific assignment weights to course directors' discretion.

\section{Considerations}

Scarcities of multiple resources are important obstacles to overcome in the implementation of an involved and multifaceted course such as PINE; we address several potential problems and workarounds here.

Financial and Human Capital: Counterintuitive to staggering tuition rates, medical student education is generally an expensive undertaking for institutions, and assembling the faculty and staff to teach an additional course could pose a considerable financial burden. Additionally, available staff, personnel, and community resources differ by institution and location. For instance, not every institution or region has easy access to a business or law professor who can lecture on bargaining, or social workers who can lecture on community outreach. Low-cost academic materials and means of training instructors and personnel would likely be essential to initiating this course. Modest volunteer stipends or gifts could be rewarded to standardized patient volunteers, guest speakers, and others. Telecommunication and existing open-access resources available online would facilitate course material development and distribution of ideas, especially in more resource-deficient locations. Such resources could include documents, Citrix ${ }^{\circledR}$ interactive webinars, texts, vignettes, video recordings of professional-led workshops or conferences, site visits, etc. 
Furthermore, existing courses that address concepts overlapping those of PINE could be adapted, modified, or reorganized for resource efficiency and enrichment.

Institutional curricular time: most medical school curriculums are already on tight schedules [23]. To not encroach on basic science lecture time and overtax students, we suggest that PINE be a once per week, 90-minute lecture preceding a 30-minute workshop, demonstration, or other in-class activity, with scheduled break(s) as needed, and mandatory attendance. Lectures may be shortened, extended, or sub-divided as needed to accommodate topic length. Videos, cases, and readings, along with relevant, brief open-ended questions, should be pre-assigned as appropriate to provide background and/or preparation for class discussions and activities. As addressed previously, grading for this type of course is recommended to be pass/fail, as most curricular time will still be devoted to basic science.

Educational Relevance: This course is fundamentally an interactive journey and its benefits to students are heavily dependent on engagement. Medical students are generally spread thin with regard to their coursework and extracurricular commitments [24], and the importance of board exam scores for residency match applications, understandably, exerts pressure on students to focus on learning topics directly "relevant" to career advancement. Given this, course staff discretion regarding when to deliver certain lectures is crucial for effective implementation and fulfillment of educational objectives. In its most effective form, this course should not be a monolithic block, but rather, a scattered seminar series, with lecture order and style at course directors' discretions. For instance, the lectures on patient interviews should be intuitively saved for periods immediately preceding clinical rotations and other patient encounters, the timing of which can differ substantially by institution. For evaluation purposes, multiple-choice, NBME $®$-style clinical vignettes could serve as a quantifiable means of assessment that also prepares students for future exam-taking.

Limitations of Human Memory: The reality of synaptic pruning is such that our retention of information is on a "use it or lose it" basis. PINE attempts to provide an active and engaging learning environment in which students can develop, reinforce, and fine-tune their skills longitudinally. Research literature supports the importance of practice in retention [25], and if teach-back is effective for patient education [26,27], it should be effective for students, as well. In addition to traditional planned redundancy and integrative lecture progression, the student group activities and evaluative teach-back presentations should provide excellent opportunities to practice and solidify the principles and skills. Even more importantly, we encourage course participants to seek out clinical and real-world opportunities to practice and apply the principles with the help of faculty and other leaders. If simulations are akin to dissector manuals, then these experiences are analogous to gross anatomy lab.

Adaptability: An essential quality of PINE is utility through flexibility. The course structure and content should be continually modified as seen appropriate and per student, faculty, and participant feedback. Further, while this course is intended for medical students, other health professionals could benefit, as well. Nurses, physician assistants, pharmacists, social workers, occupational therapists, physical therapists, and even precocious pre-health undergraduates will be working alongside physicians under similar work conditions. With the proper intra- and inter-institution cooperation and logistical coordination, this extension to wider audiences would expand PINE's impact and relevance. In the absence of a devoted nationwide network and collaborative organization that oversees and maintains a standard syllabus, PINE would likely remain provincial in reach and vary in form across locations. Nonetheless, research supports its merits and potential benefit, and implementation at even one institution would have a positive impact.

\section{Implications}

The field of medicine is constantly changing. While knowledge can become obsolete, skills are adaptable. Physicians-in-training will always need strong communication and negotiation skills to interact well with patients and providers, provide quality care, prevent and defuse conflicts, and represent the medical community effectively. We envision that our course would help healthcare professionals develop these tools needed to keep pace with future trends and catalyze better outcomes, as previous research has elaborated upon. Even a slight improvement in efficiency, diagnostic accuracy, and/or adherence from improved communication and negotiation could greatly affect outcomes, satisfaction, and cost. It is in these ways that we hope our course idea will contribute to the wellbeing of patients and society.

\section{REFERENCES}

[1] Bartlett $\mathrm{G}$ et al., Impact of patient communication problems on the risk of preventable adverse events in acute care settings. CMAJ. 2008; 178(12):1555-1562.

[2] Huntington, Beth, and Nettie Kuhn. "Communication gaffes: a root cause of malpractice claims." Proceedings (Baylor University. Medical Center) 16.2 (2003): 157

[3] Safran DG et al., Switching doctors: Predictors of voluntary disenrollment from a primary care physician's practice. J Fam Practice; 2000; 50(2):130-136.

[4] Duffy, F. D., Gordon, G. H., Whelan, G., Cole-Kelly, K., \& Frankel, R. (2004). Assessing competence in communication and interpersonal skills: The Kalamazoo II report. Academic Medicine, 79, 495-507. Pubmed

[5] Heisler, M., Bouknight, R. R., Hayward, R. A., Smith, D. M., \& Kerr, E. A. (2002). The relative importance of physician communication, participatory decision-making, and patient 
understanding in diabetes self-management. Journal of General Internal Medicine, 17, 243-252. Pubmed

[6] Renzi, C., Abeni, D., Picardi, A., Agostini, E., Melchi, C. F., Pasquini, P., Prudu, P., \& Braga, M. (2001). Factors associated with patient satisfaction with care among dermatological outpatients. British Journal of Dermatology, 145, 617-623. Pubmed

[7] Safran, D. G., Taira, D., Rogers, W. H., Kosinski, M., Ware, J. E., \& Tarlov, A. R. (1998). Linking primary care performance to outcomes of care. Journal of Family Practice, 47(3), 213-220. Pubmed

[8] Sullivan, L. M., Stein, M. D., Savetsky, J. B., \& Samet, J. H. (2000). The doctor-patient relationship and HIV-infected patients' satisfaction with primary care physicians. Journal of General Internal Medicine, 15, 462-469. Pubmed

[9] Zachariae, R., Pederson, C. G., Jensen, A. B., Ehrnrooth, E., Rossen, P. B., Von der Maase, H. (2003). Association of perceived physician communication style with patient satisfaction, distress, cancer-related self-efficacy, and perceived control over the disease. British Journal of Cancer, 88, 658-665. Pubmed

[10] Macleod, A., \& Frank, B. (2010). Patient-centredness in a context of increasing diversity: Location, location, location. Med Teacher, 799-801.

[11] Stewart, M., Brown, J., Weston, W., McWhinney, I., McWilliam, C., Freeman, T., (1995). Patient-centered medicine transforming the clinical method. London: Sage.

[12] Tsimtsiou, Z., Kerasidou, O. Efstathiou, N., Papaharitou, S., Hatzimouratidis, K., Hatzichristou, D. (2007). Medical students' attitudes toward patient-centred care: Longitudinal survey. Med Educ 41(2):140-153.

[13] Stewart, M. (1995). Effective physician-patient communication and health outcomes: a review. Canadian Medical Association Journal, 152,1423-1433.

[14] Permut SR. The difficult science you might not know--but should. AMA Wire. 23 Nov 2015, web. http://www.ama-assn.org/ama/ama-wire/post/difficult-scienc e-might-not-but-should?utm_source=BulletinHealthCare\&ut m_medium $=$ email\&utm_term $=112815 \& u$ tm_content $=$ studen ts \&utm_campaign=article_alert-morning_rounds_weekend.

[15] Malhotra D., Malhotra, M. Negotiation Strategies for Doctors - and Hospitals. Harvard Business Review. 21 Oct 2013.

Web. https://hbr.org/2013/10/negotiation-strategies-for-doctors-an d-hospitals

[16] Anastakis, Dimitri J. "Negotiation skills for physicians." The American journal of surgery 185.1 (2003): 74-78.

[17] Sarfaty, Suzanne, et al. "Negotiation in academic medicine: A necessary career skill." Journal of Women's Health 16.2 (2007): 235-244.

[18] Schwartz, Richard W., and Caroline Pogge. "Physician leadership: essential skills in a changing environment." The American journal of surgery 180.3 (2000): 187-192.

[19] Berlin, Jonathan W., and Frank J. Lexa. "Negotiation techniques for health care professionals." Journal of the American College of Radiology 4.7 (2007): 487-491.

[20] Green et al. Improving your communication skills. BMJ Careers. 25 Jan 2012, web.

http://careers.bmj.com/careers/advice/Improving_your_com $\mathrm{m}$ unication_skills

[21] Stein TS, Nagy VT, Jacobs L. Caring for patients one conversation at a time: musings from the Interregional Clinician-Patient Communication Leadership Group. Permanente J 1998 Fall;2(4):62-8

[22] Murphy M. Which of These 4 Communication Styles Are You? Forbes. 6 Aug 2015, web. https://www.forbes.com/sites/markmurphy/2015/08/06/whic h-of-these-4-communication-styles-are-you/\#3e73ee8c3adb

[23] Dotinga R. Med Schools Cut Out Cadavers. WIRED. 19 May 2003, web. https://www.wired.com/2003/05/med-schools-cut-out-cadav e rs/

[24] Klatt, Edward C., and Carolyn A. Klatt. "How much is too much reading for medical students? Assigned reading and reading rates at one medical school." Academic Medicine 86.9 (2011): 1079-1083.

[25] Caison, Amy L. "Determinants of systemic retention: Implications for improving retention practice in higher education." Journal of College Student Retention: Research, Theory \& Practice 6.4 (2005): 425-441.

[26] Tamura-Lis, Winifred. "Teach-back for quality education and patient safety." Urologic Nursing 33.6 (2013): 267.

[27] White, Matthew, et al. "Is "teach-back" associated with knowledge retention and hospital readmission in hospitalized heart failure patients?" Journal of Cardiovascular Nursing 28.2 (2013): 137-146. 